\title{
Weak antilocalization effect and high- pressure transport properties of ScPdBi single crystal
}

Cite as: Appl. Phys. Lett. 115, 172407 (2019); https://doi.org/10.1063/1.5123349

Submitted: 05 August 2019 . Accepted: 09 October 2019. Published Online: 25 October 2019

Junli Zhang (D), Zhipeng Hou, Chenhui Zhang (D), Jie Chen, Peng Li, Yan Wen, Qiang Zhang, Wenhong Wang, and Xixiang Zhang (i)
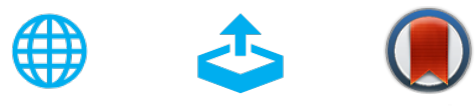

View Online

Export Citation

\section{ARTICLES YOU MAY BE INTERESTED IN}

Ferromagnetic resonance of perpendicularly magnetized $\mathrm{Tm}_{3} \mathrm{Fe}_{5} \mathrm{O}_{12} / \mathrm{Pt}$ heterostructures Applied Physics Letters 115, 172402 (2019); https://doi.org/10.1063/1.5124120

Electrical manipulation of spin pumping signal through nonlocal thermal magnon transport Applied Physics Letters 115, 172405 (2019); https://doi.org/10.1063/1.5111469

Comparative study of topological Hall effect and skyrmions in NiMnIn and NiMnGa Applied Physics Letters 115, 172404 (2019); https://doi.org/10.1063/1.5120406

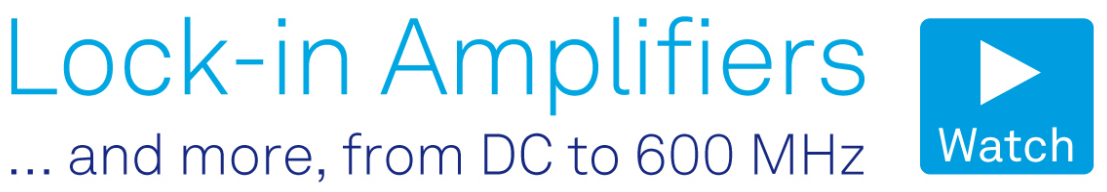




\title{
Weak antilocalization effect and high-pressure transport properties of ScPdBi single crystal
}

\author{
Cite as: Appl. Phys. Lett. 115, 172407 (2019); doi: 10.1063/1.5123349 \\ Submitted: 5 August 2019 - Accepted: 9 October 2019 . \\ Published Online: 25 October 2019
}

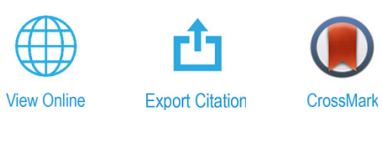

Junli Zhang, ${ }^{1}$ (D) Zhipeng Hou, ${ }^{2}$ Chenhui Zhang, ${ }^{1}$ (D) Jie Chen, ${ }^{2}$ Peng Li, ${ }^{7}$ Yan Wen, ${ }^{1}$ Qiang Zhang, Wenhong Wang, ${ }^{2, a)}$ and Xixiang Zhang, a) (iD

\begin{abstract}
AFFILIATIONS
${ }^{7}$ Division of Physical Science and Engineering (PSE), King Abdullah University of Science and Technology (KAUST), Thuwal 23955-6900, Saudi Arabia

${ }^{2}$ State Key Laboratory for Magnetism, Beijing National Laboratory for Condensed Matter Physics, Institute of Physics, Chinese Academy of Sciences, Beijing 100190, China
\end{abstract}

a) Authors to whom correspondence should be addressed: wenhong.wang@iphy.ac.cn and xixiang.zhang@kaust.edu.sa

\begin{abstract}
Half-Heusler compounds have attracted considerable attention due to their fantastic physical properties that include topological effects, Weyl fermions, unusual magnetism, and superconductivity. Herein, the transport properties of half-Heusler ScPdBi single crystals are studied across a wide temperature range and different magnetic fields. From the field-dependent magnetoresistance, we observe a clear weak antilocalization (WAL) effect below $200 \mathrm{~K}$ in the low magnetic-field region. The angle-dependent magnetoconductance and the ultralarge prefactor $\alpha$ extracted from the Hikami-Larkin-Nagaoka equation reveal that the WAL effect is a 3D bulk effect caused by strong spin-orbit coupling. We further studied the magnetotransport properties of the single crystal upon application of hydrostatic pressure and found that the energy gap of ScPdBi increases gradually as the hydrostatic pressure increases. Density functional theory calculations confirm that applying hydrostatic pressure decreases the lattice parameters and, consequently, enlarges the bandgap.
\end{abstract}

Published under license by AIP Publishing. https://doi.org/10.1063/1.5123349

Ternary half-Heusler compounds with the chemical formula $\mathrm{XYZ}$ (where $\mathrm{X}$ and $\mathrm{Y}$ are the transition or rare earth metals and $\mathrm{Z}$ is the main-group element) have attracted significant attention due to their widespread potential applications in spintronic devices, ${ }^{1,2}$ sensors, ${ }^{3}$ thermoelectric devices, ${ }^{4}$ quantum computing, ${ }^{2,5}$ and more. Since the band structures of these compounds strongly depend on the spi$\mathrm{n}$-orbit coupling (SOC) and hybridization strength, the electron properties can be finely tuned by varying the elements in these materials. ${ }^{6}$ Thus, half-Heusler compounds have become a platform for exploring novel physical properties. ${ }^{6-8}$ So far, topological phenomena, ${ }^{6-8}$ magnetism, ${ }^{9}$ superconductivity, ${ }^{5,9,10}$ weak antilocalization (WAL) effect, ${ }^{11}$ etc. have been discovered in rare-earth half-Heusler compounds XYBi (e.g., YPdBi, LuPdBi, GdPtBi, ScPtBi, and ScNiBi). ${ }^{8,11-14}$ Many theoretical studies have indicated that the indirect bandgap semiconductor ScPdBi will exhibit $s-p$ band inversion under conditions like high pressure $^{15-17}$ or when substituting Sc with other elements (e.g., Lu, Y, or Gd). ${ }^{8,11,12}$ However, this has never been studied experimentally because of the complexity in synthesizing $\mathrm{XYBi}$, where the melting point differences among the constituent elements are hard to control. Therefore, a method of preparing high-quality ScPdBi compounds is still needed, not only for exploring new physics but also for broadening the potential applications of XYBi compounds.

In this work, we synthesized high-quality $\mathrm{ScPdBi}$ single crystals and studied their transport properties and high-pressure performance in detail. We found that $\mathrm{ScPdBi}$ is a $p$-type semiconductor with a positive magnetoresistance (MR). The MR increases sharply as the magnetic field is increased up to $200 \mathrm{~K}$, which is a characteristic feature of the WAL effect. We conclude that the strong spin-orbit coupling of the bulk states is the origin of the WAL effect confirmed by the angledependent magnetoconductance and Hikami-Larkin-Nagaoka (HLN) equation, where the possible origin of the topologically protected surface state was also excluded. Moreover, we found that the energy gap of ScPdBi can be modulated by applying external hydrostatic pressure.

Single crystals of ScPdBi were grown using a Bi-flux method. ${ }^{13} \mathrm{~A}$ mixture of high purity (99.99\%) Sc, Pd, and Bi ingots with the molar ratio of 1:1:10 was placed in an alumina crucible in a glovebox filled with Ar gas. The oxygen and moisture in the glovebox were reduced below $0.5 \mathrm{ppm}$. The crucible was then sealed inside a tantalum tube under proper Ar gas pressure, followed by sealing the tantalum tube into an evacuated quartz tube. The crystal growth was carried out in a 
furnace, where the quartz tube was heated to $1150{ }^{\circ} \mathrm{C}$ at a rate of $1.5^{\circ} \mathrm{C} / \mathrm{min}$. The temperature of the furnace was kept at $1150^{\circ} \mathrm{C}$ for 72 h. Then, the tube was cooled to $650^{\circ} \mathrm{C}$ at a rate of $2{ }^{\circ} \mathrm{C} / \mathrm{h}$. Most of the excess Bi-flux was removed by centrifuging the quartz tube at $650{ }^{\circ} \mathrm{C}$, and the residual flux was finally etched using diluted hydrochloric acid. Only the appropriately shaped and sufficiently large (millimetersized) single crystals were selected for the electrical transport measurements.

The orientation of the ScPdBi single crystal was identified by the $\mathrm{X}$-ray Laue backreflection technique. The crystal structure and quality were analyzed by powder X-ray diffraction (PXRD) and transmission electron microscopy (TEM, FEI Tecnai ST), which was operated at an accelerating voltage of $300 \mathrm{kV}$. The TEM lamella was prepared using an FEI dual-beam focused ion beam-scanning electron microscope (FIB/SEM, FEI Helios G4 UX). The quantitative simulations of the atomic resolution TEM images were performed using the JEMS package. The simulation parameters were fixed as close to the experimental parameters as possible. The high voltage, $C_{s}$ coefficient, $C_{c}$ coefficient, defocus, and sample thickness were $300 \mathrm{kV}, 1.2 \mathrm{~mm}, 5 \mathrm{~mm}, 6 \mathrm{~nm}$, and $15 \mathrm{~nm}$, respectively.

To perform the electrical transport measurements, a ScPdBi single crystal was polished to $0.1 \mathrm{~mm}$ thick along the [111] direction. The sixpoint probe method was applied, with platinum wires attached to the crystal using silver epoxy as the electrical leads. The transport properties were measured using a Quantum Design physical properties measurement system (PPMS) across the temperature range of 2-300 K. To gain a deeper understanding of the high-pressure transport properties of the ScPdBi single crystal, we also studied the electronic properties using density functional theory (DFT). ${ }^{18,19}$ The value of the lattice constant is about $6.4991 \AA$ after relaxation, which is consistent with the XRD results and close to those in previous reports. ${ }^{6,16,20}$ Detailed structural information of the built prototypical model is shown in Fig. S1. The detailed structural information of the built prototypical model is shown in Fig. S1.The electrons were described at the Perdew-BurkeErnzerhof $(\mathrm{PBE})^{18}$ level of DFT implemented in the VASP package, ${ }^{21}$ and the core electrons were evaluated using projector augmented wave (PAW) methods. ${ }^{22}$ The spin-orbit coupling method ${ }^{23}$ was employed to describe the relativity effect of the heavy atoms. The Brillouin zone was sampled using a $12 \times 12 \times 12$ Monkhorst-Pack grid. The atomic structure was optimized with the energy convergence criteria of $10^{-6}$ $\mathrm{eV}$ and force convergence criteria of $0.01 \mathrm{eV} / \mathrm{A}$.

The optical photograph and X-ray Laue backreflection pattern in Figs. 1(a) and 1(b) demonstrate that the as-grown ScPdBi single crystals are multifaceted with the preferential orientation along the [111] zone axis. The chemical composition of Sc:Pd:Bi with a molar ratio of $\sim 32.7: 34.0: 33.3$ is confirmed by the EDX spectra [Fig. 1(c)] and is close to the ideal stoichiometry of ScPdBi. The powder XRD pattern of the crushed ScPdBi single crystals [Fig. 1(d)] indicates a MgAgAs-type structure with a lattice parameter of $\mathrm{a}=6.4273 \AA \mathrm{A}^{17}$ The highresolution TEM (HRTEM) image and SAED pattern of the [111] zone axis [Figs. $1(\mathrm{e})-1(\mathrm{~g})$ ] confirm that there is no visible amorphous region or stacking faults in the sample. In addition, the consistency of the atomic columns when contrasting the simulated HRTEM image [Fig. 1(f)] of an ideal ScPdBi compound with the experimental image further indicates the high quality of the ScPdBi single crystal.

Figure 2(a) shows the temperature dependence of the longitudinal resistivity $\rho_{x x}$ measured from $2 \mathrm{~K}$ to $300 \mathrm{~K}$ under zero magnetic
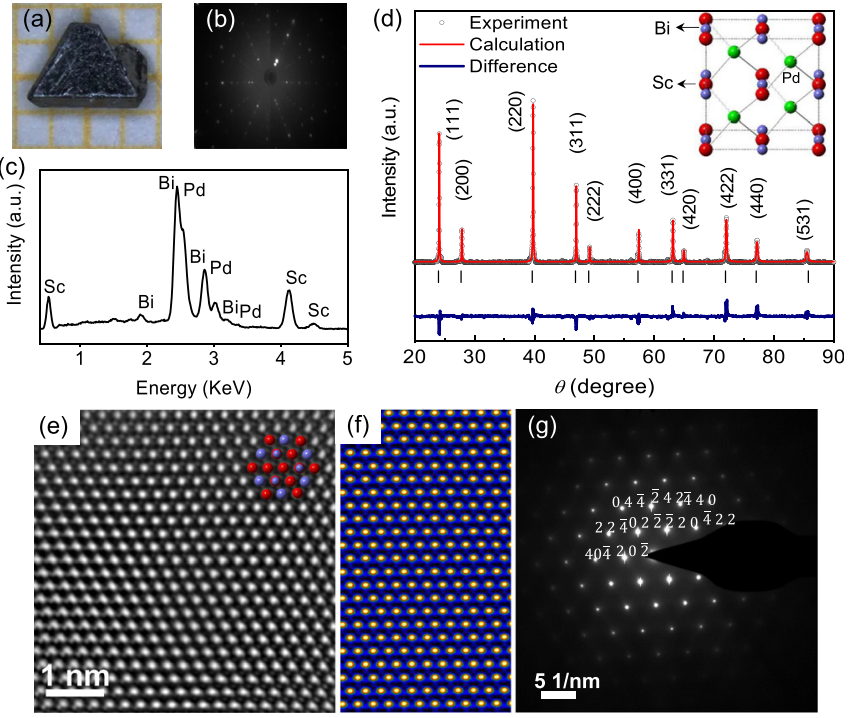

FIG. 1. (a) Optical image of a ScPdBi single crystal. (b) Laue backreflection pattern for an incident beam parallel to the [111] zone axis; all the reflections can be indexed to face-centered cubic ScPdBi. (c) Energy-dispersive X-ray (EDX) spectra and (d) powder XRD pattern of the as-prepared ScPdBi single crystal. The inset of (d) illustrates the crystal structure of ScPdBi with four formula units per unit cell. (e) and $(f)$ Experimental and theoretical high-resolution TEM images along the [111] zone axis of ScPdBi. (g) Selected area electron diffraction (SAED) pattern of $\mathrm{ScPdBi}$ along the [111] zone axis.
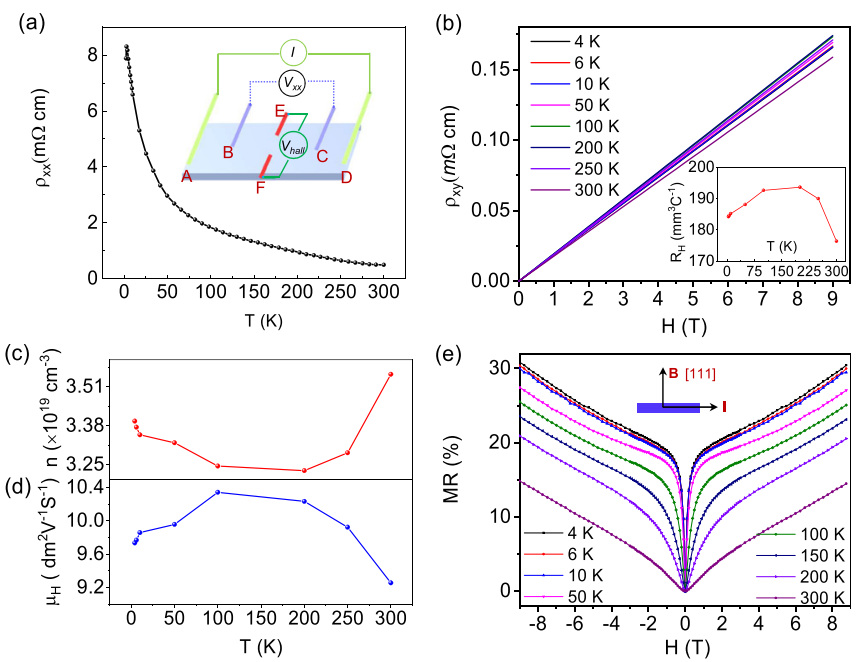

FIG. 2. (a) Temperature dependence of the longitudinal resistivity $\rho_{x x}$ under zero magnetic field. (b) Field dependence of the Hall resistivity $\rho_{x y}$ in the temperature range of $4-300 \mathrm{~K}$. The inset shows the temperature-dependent Hall coefficient $R_{H}$. (c) and (d) Temperature dependence of the carrier concentration $n$ and mobility $\mu_{H}$. (e) Normalized magnetoresistivity (MR) at temperatures ranging from 4 to $300 \mathrm{~K}$. The MR is defined as MR $=\frac{\rho_{x x}(H)-\rho_{x x}(0)}{\rho_{x x}(0)} \times 100 \%$, where $\rho_{x x}(H)$ and $\rho_{x x}(0)$ are the longitudinal resistivities measured at field $\mathrm{H}$ and zero field, respectively. The measured MR curves are symmetrized via $M R(H)=[M R(H)+M R(-H)] / 2$ to remove the Hall contribution. The inset displays a schematic arrangement of the magnetic field and current applied to the sample, where the magnetic field is applied from $-9 \mathrm{~T}$ to $9 \mathrm{~T}$. 
field. The inset of Fig. 2(a) shows a schematic of the measurement configuration, in which electrodes A, B, C, and D are used for the longitudinal MR measurements and the electrodes $\mathrm{A}, \mathrm{D}, \mathrm{E}$, and $\mathrm{F}$ are for the Hall measurements. The negative slope of $\rho_{x x}$ over the whole temperature range and the positive slopes of the Hall resistivity $\rho_{x y}$ in Fig. 2(b) indicate that the $\mathrm{ScPdBi}$ compound is a $p$-type semiconductor. The carrier concentration $\left(n_{h}\right)$ and Hall mobility $\left(\mu_{H}\right)$ are also extracted from the $\rho_{x y}$ data based on the single-band model, as shown in Figs. 2 (c) and $2(\mathrm{~d})$. The carrier concentration ranges between $3.23 \times 10^{19}$ $\mathrm{cm}^{-3}$ and $3.55 \times 10^{19} \mathrm{~cm}^{-3}$ [Fig. 2(c)]; these values are on the same order as those obtained from other rare-earth half-Heusler compounds. ${ }^{13}$ The temperature dependence of the Hall mobility, which ranges from $925 \mathrm{~cm}^{2} / \mathrm{V} \mathrm{s}$ to $1038 \mathrm{~cm}^{2} / \mathrm{V} \mathrm{s}$, is comparable to that of $\mathrm{ScNiBi}$ but lower than that of ScPtBi, LuPtBi, and YPtBi.

The mobility of the charge carriers in ScPdBi should be determined by the ionized impurity ${ }^{24}$ and lattice vibration, ${ }^{25}$ where the grain boundary scattering can be neglected due to the high quality of the single crystal. The lattice vibration arises from the heat or thermal energy in the lattice and becomes more obvious at elevated temperatures. The ionized impurity scattering derives from the crystal impurity and increases when the temperature decreases. ${ }^{24}$ This behavior is further confirmed by the positive and negative temperature dependences of $\mu_{H}$ within the temperature ranges of $4-200 \mathrm{~K}$ and above $200 \mathrm{~K}$, respectively. Figure 2(e) shows the MR measured at different temperatures with a magnetic field applied perpendicular to the (111) plane. The MR is positive over the whole temperature range $(4-300 \mathrm{~K})$ and magnetic field range ( $-9 \mathrm{~T}$ to $9 \mathrm{~T}$ ) and decreases with increasing temperature [as shown in Fig. 2(e)]. A positive MR up to 31\% is observed at $4 \mathrm{~K}$ under a magnetic field of $9 \mathrm{~T}$. The most intriguing feature in the MR curves [Fig. 2(e)] is the cusplike, strongly positive MR in the low magnetic field $(|\mathrm{H}|<0.8 \mathrm{~T})$ range at temperatures below $200 \mathrm{~K}$. This weak antilocalization (WAL) effect originates from the topologically protected surface state ${ }^{26}$ or the destructive interference of a rotated spin due to the presence of strong spin-orbit coupling ${ }^{27}$ of the 3D bulk state. It has been observed in topological insulators $\left(\mathrm{Sb}_{2} \mathrm{Te}_{3},{ }^{28} \mathrm{Bi}_{2} \mathrm{Se}_{3}{ }^{26}\right.$ $\mathrm{Bi}_{2} \mathrm{Te}_{3}{ }^{29}$ etc.), topological semimetal $\left(\mathrm{LuPdBi}^{11}\right)$, and topological superconductors ( $\mathrm{Al}-\mathrm{InAs} \mathrm{s}^{30}$ and $\mathrm{LuPdBi}{ }^{11}$ ). In addition, this phenomenon was also observed in systems with strong spin-orbit coupling, such as Sb films, $\mathrm{Na}_{2} \mathrm{IrO}_{3}$ films, ${ }^{31}$ and $\mathrm{Mg}$ films covered by $\mathrm{Au}$, LuPtSb single crystals, ${ }^{33}$ etc.

To figure out the origin of the WAL effect in the case of the ScPdBi single crystal, we study the WAL effect by fitting the low field magnetoconductance using the Hikami-Larkin-Nagaoka (HLN) equation $^{34}$ for a two-dimensional (2D) system with strong spin-orbit coupling. In this model, the magnetoconductance is formulated as

$$
\Delta G(H)=\frac{\alpha e^{2}}{2 \pi^{2} \hbar}\left[\psi\left(\frac{1}{2}+\frac{\hbar}{4 e H L_{\varphi}}\right)-\ln \left(\frac{\hbar}{4 e H L_{\varphi}}\right)\right],
$$

where $e, \hbar, \psi$, and $L_{\varphi}$ are the electron charge, reduced plank constant, digamma function, and phase coherence length, respectively. The coefficient $\alpha$ denotes the number of conduction channels, which is theoretically predicted to be -0.5 for one conduction channel and -1 for a topological insulator because the surface state exists on its both sides. Figure 3(a) displays the low-field magnetoconductance $\Delta G[\Delta G(H)$ $=G(H)-G(0)]$ at temperatures ranging from 4 to $50 \mathrm{~K}$ (dashed line), which can be described well by the HLN formula (solid black line). The fitted coherence length $L_{\varphi}$ and coefficient $\alpha$ are presented in
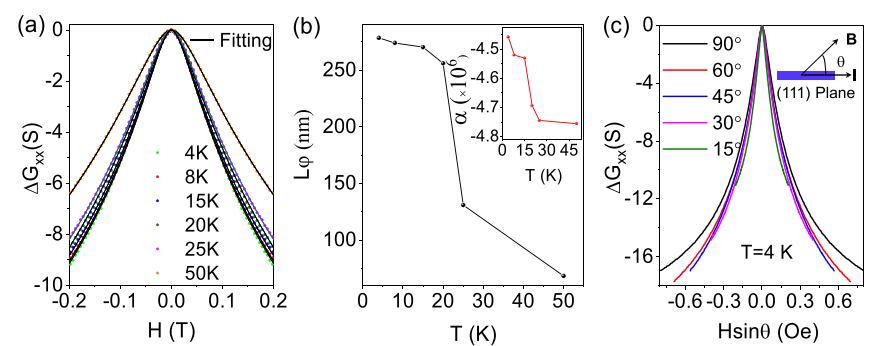

FIG. 3. (a) Magnetoconductance curves of the ScPdBi single crystal at various temperatures. (b) Fitted phase coherence length $L_{\varphi}$ and prefactor $\alpha$ in a lowtemperature regime. (c) Magnetoconductance curves as a function of $H \sin \theta$ measured at $4 \mathrm{~K}$. The inset shows a schematic of the measurement configuration, where $\theta$ indicates the angle between magnetic field $\mathrm{H}$ and the (111) plane.

Fig. 3(b). However, the values of $\alpha$ are on the order of $10^{6}$, which are much larger than -0.5 and have no physical meaning, hinting that the WAL effect in the ScPdBi crystal mainly originates from the strong spin-orbit coupling of the $3 \mathrm{D}$ bulk state. This point is further supported by the angle-dependent $\Delta G(H)$ at $4 \mathrm{~K}$ under low magnetic fields $(-0.8 \mathrm{~T}$ to $0.8 \mathrm{~T})$, as shown in Fig. $3(\mathrm{c})$. We find that the WAL can also be observed when the magnetic field is in the (111) plane (i.e., $\theta=0^{\circ}$ ). This result demonstrates that the origin of the observed WAL effect is from the $3 \mathrm{D}$ bulk states. ${ }^{35}$ Note that the origin of such a WAL effect is different from those observed in 2D topological insulators, which is always caused by the topologically protected surface states and depends on the thickness of the sample. Thus, the WAL effect can be observed in one-, ${ }^{36}$ two-, ${ }^{26,37}$ or three- ${ }^{33,37}$ dimensional materials. Besides, the deviations of the $\Delta G(H)$ vs. $H \sin \theta$ curves at different angles further reinforce that the $3 \mathrm{D}$ bulk state is the underlying physical origin of the observed WAL, rather than the possibility of the $2 \mathrm{D}$ nature because of the absence of typical overlap phenomena. ${ }^{29}$

To gain further insight into the physical properties of the ScPdBi single crystal, the transport properties were studied under different hydrostatic pressures. Figure 4(a) shows the temperature dependence of the zero-field $\rho_{x x}$ measured under various pressures. The ScPdBi single crystal maintained its semiconducting behavior throughout the pressure range, from $0 \mathrm{GPa}$ to $1.65 \mathrm{GPa}$, although the sample became more resistive across the whole temperature range with increasing pressure. To better visualize the $\rho_{x x}$-T behavior at low temperatures, a semilog plot is shown in the inset of Fig. 4(a). The influence of temperature on the resistivity in semiconductors can be described by the Arrhenius equation, ${ }^{14,38}$

$$
\rho=\rho_{0} \exp \left(\mathrm{Ea} / 2 k_{B} T\right) .
$$

According to Eq. (2), the activation energy $E_{a}$ of $\mathrm{ScPdBi}$ is calculated by linearly fitting $\ln \rho$ vs $1 / T$ [Fig. 4(b)]. As shown in the inset of Fig. 4(b), the value of $E_{a}$ increases monotonically as the pressure increases at a rate of $3.58 \mathrm{meV} / \mathrm{GPa}$. The relationship between the transport properties of the $\mathrm{ScPdBi}$ single crystal and hydrostatic pressure was interpreted using DFT computations. ${ }^{16}$ The calculated electronic band structures of ScPdBi under different hydrostatic pressures are shown in Figs. 4(c) and 4(d), in which an indirect bandgap between the conduction and valence bands can be observed. Besides, the band structures without spin-orbit coupling (SOC) are also studied in Fig. S3 for comparison. We find that the bottom conduction band splits into the 

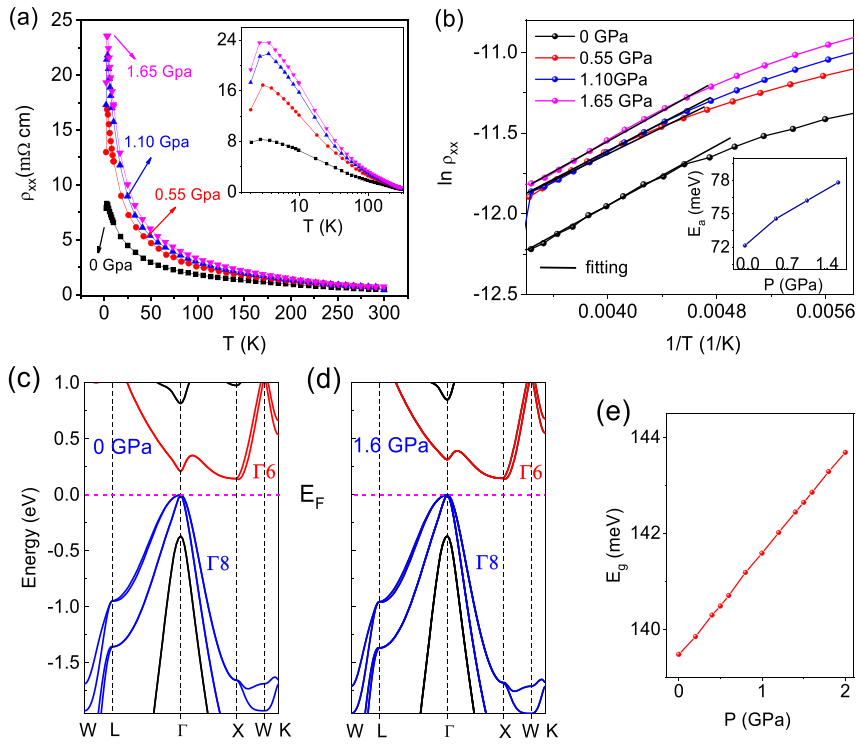

FIG. 4. (a) Longitudinal resistivity $\rho_{x x}$ under different hydrostatic pressures. The inset shows a semilog plot of $\rho_{x x}$ vs T. (b) Arrhenius plots of the ScPdBi single crystal under varying hydrostatic pressures. The inset shows the summarized activation energy as a function of pressure, fitted by the Arrhenius equation. (c) and (d) Electronic band structures of $\mathrm{ScPdBi}$ at $0 \mathrm{GPa}$ and $16 \mathrm{GPa}$, respectively. (e) Calculated energy gap of ScPdBi under different pressures.

$\Gamma_{6}$ state that has $s$-like symmetry with twofold degeneracy and also the top valence band splits into $\Gamma_{8}$ states ( $p$-like symmetry) with fourfold degeneracy when the SOC was considered. The energy difference between $\Gamma_{6}$ and $\Gamma_{8}$ states (i.e., band inversion strength $\Delta E=E_{\Gamma 6}$ $-E_{\Gamma 8}$ ) can be used to judge the topological state of material. ${ }^{2,17}$ In general, negative and positive $\Delta E$ correspond to the topologically nontrivial and trivial phase, respectively. The positive signs of $\Delta \mathrm{E}$ obtained from Figs. 4(c) and 4(d) show the absence of $s / p$ inversion (i.e., the feature of the topological insulator). These features demonstrate that $\mathrm{ScPdBi}$ is a trivial semiconductor, and there is no topological phase transition observed when the pressure changes from $0 \mathrm{GPa}$ to $1.6 \mathrm{GPa}$. In particular, the simulated positive dependence of the energy gap on pressure [shown in Fig. 4(e)] is the same as the experimental trend in the activation energy and resistivity variation [inset of Fig. 4(b)]. The variation of the direct bandgap at the $\Gamma$ point (Fig. S3) shows the same trend with pressure but at a faster rate. This is because applying hydrostatic pressure decreases the lattice parameter of $\mathrm{ScPdBi}$, which results in a larger bandgap. Herein, we think that the origin of the change in the bandgap is due to the change in lattice parameters. Note that the deviation can also occur such as in topological insulators when the pressure was applied. This has been demonstrated by the change in the Berry phase, Fermi pockets, and band inversion state. ${ }^{39-41}$ However, such deviations caused by the topologically protected crossings and band inversion (i.e., caused by the spin-orbit coupling) can be ignored here due to the trivial semiconductor characteristics of ScPdBi. The consistency between the experimental and theoretical results confirms the tunability of the electronic transport properties in a ScPdBi single crystal by external hydrostatic pressure.

In conclusion, ScPdBi was confirmed to be a trivial semiconductor with a positive MR. An interesting cusplike WAL feature was observed in the low magnetic-field region of the MR curves at temperatures below $200 \mathrm{~K}$. As demonstrated by the unphysical value of the fitting coefficient $\alpha$ and the angle dependence of the $\Delta G(H)$ curves, the origin of the WAL was the strong spin-orbit coupling of the $3 \mathrm{D}$ bulk state. An investigation of the effect of hydrostatic pressure on the transport properties further indicated the feasibility of modulating the electronic state of the ScPdBi single crystal.

See the supplementary material for the calculated prototypical ScPdBi structure, electronic band structures of ScPdBi without spi$\mathrm{n}$-orbit coupling at $0 \mathrm{Gpa}$ and $16 \mathrm{GPa}$, and calculated direct bandgap of ScPdBi under different pressures.

This work was supported by the King Abdullah University of Science and Technology (KAUST), Office of Sponsored Research (OSR) under Award No. CRF-2015-2549-CRG4, and China Postdoctoral Science Foundation No. Y6BK011M51.

\section{REFERENCES}

${ }^{7}$ F. Casper, T. Graf, S. Chadov, B. Balke, and C. Felser, Semicond. Sci. Technol. 27, 063001 (2012).

${ }^{2}$ W. X. Feng, D. Xiao, Y. Zhang, and Y. G. Yao, Phys. Rev. B 82, 235121 (2010).

${ }^{3}$ R. Y. Umetsu, K. Kobayashi, A. Fujita, K. Oikawa, R. Kainuma, K. Ishida, N. Endo, K. Fukamichi, and A. Sakuma, Phys. Rev. B 72, 214412 (2005).

${ }^{4}$ W. G. Zeier, J. Schmitt, G. Hautier, U. Aydemir, Z. M. Gibbs, C. Felser, and G. J. Snyder, Nat. Rev. Mater. 1, 16032 (2016).

${ }^{5}$ H. Lin, L. A. Wray, Y. Xia, S. Xu, S. Jia, R. J. Cava, A. Bansil, and M. Z. Hasan, Nat. Mater. 9, 546-549 (2010).

${ }^{6}$ S. Chadov, X. Qi, J. Kubler, G. H. Fecher, C. Felser, and S. C. Zhang, Nat. Mater. 9, 541-545 (2010).

${ }^{7}$ D. Xiao, Y. Yao, W. Feng, J. Wen, W. Zhu, X. Q. Chen, G. M. Stocks, and Z. Zhang, Phys. Rev. Lett. 105, 096404 (2010).

${ }^{8}$ M. Hirschberger, S. Kushwaha, Z. Wang, Q. Gibson, S. Liang, C. A. Belvin, B. A. Bernevig, R. J. Cava, and N. P. Ong, Nat. Mater. 15, 1161-1165 (2016).

${ }^{9}$ B. R. K. Nanda and I. Dasgupta, J. Phys.: Condens. Matter 15, 7307-7323 (2003).

${ }^{10}$ N. P. Butch, P. Syers, K. Kirshenbaum, A. P. Hope, and J. Paglione, Phys. Rev. B 84, 220504 (2011).

${ }^{11} \mathrm{G}$. Xu, W. Wang, X. Zhang, Y. Du, E. Liu, S. Wang, G. Wu, Z. Liu, and X. X. Zhang, Sci. Rep. 4, 5709 (2014).

${ }^{12}$ W. Wang, Y. Du, G. Xu, X. Zhang, E. Liu, Z. Liu, Y. Shi, J. Chen, G. Wu, and X. X. Zhang, Sci. Rep. 5, 7941 (2015).

${ }^{13}$ Z. P. Hou, Y. Wang, E. K. Liu, H. W. Zhang, W. H. Wang, and G. H. Wu, Appl. Phys. Lett. 107, 202103 (2015).

${ }^{14}$ L. Deng, Z. H. Liu, X. Q. Ma, Z. P. Hou, E. K. Liu, X. K. Xi, W. H. Wang, G. H. $\mathrm{Wu}$, and X. X. Zhang, J. Appl. Phys. 121, 105106 (2017).

${ }^{15}$ M. Narimani and Z. Nourbakhsh, Thin Solid Films 616, 287-296 (2016).

${ }^{16}$ M. Narimani and Z. Nourbakhsh, Mod. Phys. Lett. B 30, 1650159 (2016).

${ }^{17}$ W. Al-Sawai, H. Lin, R. S. Markiewicz, L. A. Wray, Y. Xia, S. Y. Xu, M. Z. Hasan, and A. Bansil, Phys. Rev. B 82, 125208 (2010).

${ }^{18}$ J. P. Perdew, K. Burke, and M. Ernzerhof, Phys. Rev. Lett. 77, 3865-3868 (1996).

${ }^{19}$ P. Geerlings, F. De Proft, and W. Langenaeker, Chem. Rev. 103, 1793-1873 (2003).

${ }^{20}$ X. Zhang, Z. Hou, Y. Wang, G. Xu, C. Shi, E. Liu, X. Xi, W. Wang, G. Wu, and X. X. Zhang, Sci. Rep. 6, 23172 (2016)

${ }^{21}$ G. Kresse and J. Furthmuller, Phys. Rev. B 54, 11169-11186 (1996).

${ }^{22}$ P. E. Blochl, Phys. Rev. B 50, 17953-17979 (1994).

${ }^{23}$ S. Steiner, S. Khmelevskyi, M. Marsmann, and G. Kresse, Phys. Rev. B 93, 224425 (2016).

${ }^{24}$ E. Conwell and V. F. Weisskopf, Phys. Rev. 77, 388-390 (1950).

${ }^{25}$ C. Herring and E. Vogt, Phys. Rev. 101, 944-961 (1956).

${ }^{26}$ J. Chen, H. J. Qin, F. Yang, J. Liu, T. Guan, F. M. Qu, G. H. Zhang, J. R. Shi, X. C. Xie, C. L. Yang, K. H. Wu, Y. Q. Li, and L. Lu, Phys. Rev. Lett. 105, 176602 (2010). 
${ }^{27}$ G. Bergmann, Phys. Rep. 107, 1-58 (1984).

${ }^{28}$ Y. Takagaki, A. Giussani, K. Perumal, R. Calarco, and K. J. Friedland, Phys. Rev. B 86, 125137 (2012).

${ }^{29}$ H. T. He, G. Wang, T. Zhang, I. K. Sou, G. K. Wong, J. N. Wang, H. Z. Lu, S. Q. Shen, and F. C. Zhang, Phys. Rev. Lett. 106, 166805 (2011).

${ }^{30}$ A. Das, Y. Ronen, Y. Most, Y. Oreg, M. Heiblum, and H. Shtrikman, Nat. Phys. 8, 887-895 (2012).

${ }^{31}$ M. Jenderka, J. Barzola-Quiquia, Z. P. Zhang, H. Frenzel, M. Grundmann, and M. Lorenz, Phys. Rev. B 88, 045111 (2013).

${ }^{32}$ G. Bergman, Phys. Rev. Lett. 48, 1046-1049 (1982).

${ }^{33}$ Z. P. Hou, Y. Wang, G. Z. Xu, X. M. Zhang, E. K. Liu, W. Q. Wang, Z. Y. Liu, X. K. Xi, W. H. Wang, and G. H. Wu, Appl. Phys. Lett. 106, 102102 (2015).

${ }^{34}$ S. Hikami, A. I. Larkin, and Y. Nagaoka, Prog. Theor. Phys. 63, 707-710 (1980).
35. Rammer, Quantum Transport Theory (Perseus Books, 1998).

${ }^{36}$ J. Kim, S. Lee, Y. M. Brovman, M. G. Kim, P. Kim, and W. Lee, Appl. Phys. Lett. 104, 043105 (2014).

${ }^{37}$ Y. S. Kim, M. Brahlek, N. Bansal, E. Edrey, G. A. Kapilevich, K. Iida, M. Tanimura, Y. Horibe, S.-W. Cheong, and S. Oh, Phys. Rev. B 84, 073109 (2011).

${ }^{38}$ K. J. Laidler, J. Chem. Educ. 61, 494-498 (1984).

${ }^{39}$ J. L. Zhang, C. Y. Guo, X. D. Zhu, L. Ma, G. L. Zheng, Y. Q. Wang, L. Pi, Y. Chen, H. Q. Yuan, and M. L. Tian, Phys. Rev. Lett. 118, 206601 (2017).

${ }^{40}$ Z. J. Xiang, G. J. Ye, C. Shang, B. Lei, N. Z. Wang, K. S. Yang, D. Y. Liu, F. B. Meng, X. G. Luo, L. J. Zou, Z. Sun, Y. Zhang, and X. H. Chen, Phys. Rev. Lett. 115, 186403 (2015).

${ }^{41}$ P. L. Cai, J. Hu, L. P. He, J. Pan, X. C. Hong, Z. Zhang, J. Zhang, J. Wei, Z. Q. Mao, and S. Y. Li, Phys. Rev. Lett. 115, 057202 (2015). 